\title{
Circuit
}

Musiques contemporaines

CIRCUIT

\section{Le circuit du désir musical}

L'interprète, le compositeur, l'auditeur — organes et instruments

\section{The Circuit of Musical Desire}

\section{The Performer, the Composer, the Audience - Organs and Instruments}

\section{Bernard Stiegler et Nicolas Donin}

Volume 15, numéro 1, 2004

Interpréter la musique (d')aujourd'hui

URI : https://id.erudit.org/iderudit/902340ar

DOI : https://doi.org/10.7202/902340ar

Aller au sommaire du numéro

\section{Éditeur(s)}

Les Presses de l'Université de Montréal

ISSN

1183-1693 (imprimé)

1488-9692 (numérique)

Découvrir la revue

Citer ce document

Stiegler, B. \& Donin, N. (2004). Le circuit du désir musical : l'interprète, le compositeur, l'auditeur — organes et instruments. Circuit, 15(1), 41-56. https://doi.org/10.7202/902340ar
Résumé de l'article

Partant d'une déconstruction de l'opposition entre production et reproduction qui sous-tend le couple compositeur / interprète, Bernard Stiegler propose dans cet entretien de penser la relation entre musiciens et auditeurs comme un circuit constitué par un tissu d'exclamations - relevant à ce titre d'une même logique d'interprétation, c'est-à-dire de reproduction des singularités.

L'entretien précise comment articuler dans ce cadre les principaux concepts philosophiques forgés par Stiegler à partir de Husserl et de Simondon : rétentions primaires, secondaires, secondaires collectives, tertiaires; relation transductive et processus d'individuation; épiphylogénèse et système technique.

Réciproquement, la musique donne à la philosophie la base pour fonder, en référence notamment au projet généalogique nietzschéen, une organologie générale qui permettrait de repenser l'esthétique humaine dans toute son historicité, en articulant étroitement organes des sens, prothèses et instruments, organismes et institutions. 


\section{Le circuit du désir musical}

\section{L'interprète, le compositeur, l'auditeur - organes et instruments}

Bernard Stiegler

Entretien avec Nicolas Donin'

NiColas Donin : Il se cache souvent, derrière les comparaisons ou les oppositions entre interprète et compositeur, un antagonisme plus profond : l'interprète serait reproducteur et le compositeur, producteur. Reprendrais-tu ces termes si tu devais à ton tour les définir l'un par rapport à l'autre?

BERNARD STIEGLER : est avant toute autre chose un interprète qui, parfois, ne se sait pas interprète. Il faut distinguer l'interprète de l'instrumentiste. Si le compositeur n'a pas affaire à un instrument, il est cependant inscrit dans une organologie musicale que l'on a (bien à tort) réduite jusqu'ici à celle des instrumentistes - à ce que l'on appelle en général la lutherie. L'écriture, dans le sens que je tente de conférer à l'organologie (que je dis alors «élargie»), fait partie d'un système technique de la musique dont les instruments ne sont que des aspects et bien sûr, les tout premiers aspects. Le compositeur a des techniques d'écriture, des règles d'écriture, il est inscrit dans une histoire de l'écriture constituée de textes qu'il interprète, et qui hantent ses oreilles comme attentes d'un avenir de la musique, tout entières tramées par le passé de la musique. À partir d'une certaine époque, ces attentes peuvent devenir celles de ruptures, inscrites cependant dans ce que j'appelle, avec Simondon, un processus d'individuation psychique et collective. Ces attentes, ces expectations au sens de Leonard B. Meyer, ce ne sont pas simplement des attentes individuelles : ce sont celles d'une époque. C'est en cela qu'il faut élargir la pensée de Meyer, par exemple, aux expectations
1. Ce texte a été réalisé, à partir d'un entretien par courriels, de février à mai 2004 . 
2. Strette (2003) pour soprano, électronique et vidéo en temps réel. d'un nous musical, un peu comme Bernard Lortat-Jacob tente de le penser avec la musique sarde. Quant au compositeur, il a accès à des corpus de «littérature » musicale dans les conditions non temporelles et spatialisées qu'a si bien décrites Hugues Dufourt, en sorte qu'il constitue non seulement un savoir de la musique, mais bien une connaissance de celle-ci. C'est en cela que l'on aurait tendance à éliminer la dimension interprétative, on pourrait être tenté de voir dans ce que l'on appelle l'écriture savante de la musique un processus de découverte plutôt que d'interprétation, et, en cela, de libération de l'histoire. Cependant, un tel point de vue est une illusion : lorsqu'il écrit, qu'il le sache ou pas, un compositeur ne fait qu'écrire ce qu'il a entendu, et il ne s'agit d'ailleurs pas nécessairement de ce qu'il a entendu dans la musique savante, ni même dans la musique. Un musicien, c'est quelqu'un qui d'abord entend, c'est-à-dire qu'il est primordialement affecté par l'oreille, une oreille qui a cependant des yeux et des mains, et un corps qui les relie. Il ne se contente pas de calculer. Il peut calculer, il doit même calculer, mais s'il le fait, c'est pour donner à entendre ce qu'il a lui-même entendu comme l'incalculable même.

Ceci s'inscrit dans un circuit du désir qui se constitue comme un tissu d'exclamations. Ces exclamations sont toujours à retardement, elles sont les échos plus ou moins lointains, par exemple, de Monteverdi, Mozart, Beethoven, Mahler, Webern ou Stravinsky, qui sont autant de coups reçus par une oreille qui les rend en composant, des " coups de boutoir dans tous les sens» comme disait Artaud, et ces contre-dons peuvent avoir lieu à l'occasion d'autres coups, d'autres chocs, d'autres affects qui ne sont pas nécessairement de nature musicale : une femme, un sourire, un poème de Celan, comme dans une pièce récente d'Hector Parra ${ }^{2}$, le cinéma, comme chez Olga Neuwirth. Ce peut être aussi une inspiration musicale radicalement non savante, comme Aphex Twin pour Andrea Cera. Lorsque le compositeur écoute ou regarde ou lit quelque chose, par exemple, ça se traduit par des choses qu'il entend, ainsi de Debussy quant à L'après-midi d'un faune. Cette «traduction » peut avoir lieu très en différé, comme le dit Klee à propos de sa peinture comme mémoire tardive du voir. Mais toute sensibilité noétique (c'est ainsi qu'Aristote caractérise les âmes des humains) est ainsi affectée, et elle ne constitue son affect qu'en l'extériorisant dans un circuit qui est aussi une "différance», au sens de Derrida. Cependant, le compositeur le fait depuis les affections primaires (pour lui en tant que musicien) qu'auront été dans la constitution de son oreille les œuvres musicales écrites qu'il a étudiées et qu'il interprète, au-delà de la nécessaire connaissance qui lui permet de «s'exprimer ", c'est-à-dire ici d'écrire et de composer. Le compositeur a donc un organe auditif ou, disons, un appareil d'écoute, qui a besoin de se traduire sous la forme de ce que j'appelle, dans un 
texte que je viens d'écrire ${ }^{3}$, la forme exclamative, c'est-à-dire qu'il a besoin de spatialiser et de temporaliser son écoute, en sorte que celle-ci devient immédiatement une écriture. J'ai dit ailleurs que lire c'était écrire, et qu'écrire c'était toujours lire. De même, Cézanne dit de la montagne qu'il ne la voit que dans la mesure où il est capable de la montrer. Schubert, Pauset l'entend dans la mesure où il le donne à entendre, ce qui est aussi une démesure.

Quant à l'interprète, au sens habituel du terme, c'est-à-dire l'instrumentiste, ce n'est pas quelqu'un qui reproduit. L'interprète ne reproduit rien, sauf à dire que toute production, toute création, toute com-position est toujours déjà une repro-duction. Non pas une re-production qui supposerait qu'il y a d'abord une production puis ensuite une reproduction, mais bien une repro-duction où la «duction » est ce que donne la répétition, une répétition originaire : il n'y a pas de musique, il n'y a pas d'art en général hors d'un horizon de répétition. Cet horizon de répétition est, par exemple, la répétition de la gamme que le pianiste ou l'instrumentiste fait sur son instrument, c'est la répétition des tonalités, ou des règles d'atonalité, et c'est l'ensemble extrêmement riche et varié des rétentions secondaires collectives qui caractérisent l'histoire et la géographie de la musique mondiale.

La rétention et la protention sont les concepts par lesquels Husserl spécifie l'objet temporel qu'est, par exemple, une mélodie. Un tel objet temporel est constitué par le temps de son écoulement, que Husserl nomme son flux. Il n'apparaît qu'en disparaissant : c'est un objet qui passe. La conscience est également temporelle en ce sens. Un objet temporel est constitué par le fait que, comme les consciences dont il est l'objet commun, il s'écoule et disparaît à mesure qu'il apparaît. Un « je» est une conscience consistant en un flux temporel de ce que Husserl appelle des «rétentions primaires» : la rétention primaire est ce que la conscience retient dans le maintenant du flux en quoi elle consiste. C'est, par exemple, la note qui résonne dans une note présente à ma conscience comme point de passage d'une mélodie, et où la note précédente n'est pas absente, mais bien présente, parce que maintenue dans et par le maintenant : elle constitue la note qui la suit en formant avec elle un rapport, l'intervalle. C'est aussi le mot que je viens de prononcer ou d'écrire et qui retient primairement le mot qui le précède pour constituer le sens d'une phrase, qui retient elle-même la phrase précédente pour constituer l'unité de mon discours, etc. Comme phénomènes que je reçois aussi bien que comme phénomènes que je produis (une mélodie que je joue ou entends, une phrase que je prononce ou entends, une séquence de gestes ou d'actions que j'accomplis ou que je subis, etc.), ma vie consciente consiste essentiellement en de telles rétentions. Or, ces rétentions sont des sélections : je ne retiens pas tout ce qui peut
3. "La conjonction qui disjoncte dans les tourbillons de la nécessité", La sœur de l'Ange, $n^{\circ} 1$, avril 2004 
4. Les rétentions primaires forment des relations : par exemple, dans une œuvre musicale, des enchaînements harmoniques; ou dans une phrase, des liens sémantiques et syntaxiques. être retenu ${ }^{4}$. Dans le flux de ce qui apparaît, la conscience opère des sélections qui sont les rétentions en propre : si j'écoute deux fois de suite la même mélodie, ma conscience de l'objet change. Ces sélections se font à travers les filtres en quoi consistent les rétentions secondaires, c'est-à-dire les souvenirs de rétentions primaires antérieures, que conserve la mémoire, qui constituent l'expérience, et au sein desquelles se forment des protentions, des expectations de la conscience. La vie de la conscience consiste en de tels agencements de rétentions primaires, filtrées par des rétentions secondaires, formant autant de protentions, tandis que les rapports des rétentions primaires et secondaires sont surdéterminés par les rétentions tertiaires : les objets supports de mémoire et les mnémotechniques, qui permettent d'enregistrer spatialement, matériellement et techniquement des traces. Les rétentions tertiaires sont des supports de répétitions (qu'il s'agisse d'instruments de musique, de partitions, de bandes magnétiques, de disques ou de fichiers numériques et d'algorithmes, de programmes) où se produit une repro-duction, c'est-à-dire où il apparaît que toute rétention primaire, en tant qu'elle mobilise une rétention secondaire qui vient la sélectionner et qui donc la précède, n'est jamais une pure production, mais bien une telle repro-duction.

C'est dans cette situation que se forment des rétentions secondaires collectives, des horizons d'attentes typiques de telles cultures musicales, qui préparent les oreilles, en sorte que les oreilles, au-delà de leurs singularités individuelles, attendent ensemble quelque chose de ce qu'elles entendent, dans ce qu'elles entendent, dans tout ce qu'elles entendent. Notre oreille occidentale est préconstituée par ces attentes qui sont liées à des rétentions tertiaires qui nous sont propres, telle la partition. Quant à l'interprète, c'est quelqu'un qui repro-duit, tout comme le compositeur repro-duit. Instrumentistes et compositeurs sont dans la repro-duction qu'ils interprètent chaque fois singulièrement.

N. D. : La distinction entre interprète et compositeur n'a pas toujours existé dans l'histoire de la musique occidentale. Sa fortune et son maintien semblent avoir tenu précisément à cette répartition des rôles, en apparence très tranchée, qui voulait que les uns produisent ce que les autres reproduiraient. Mais cette dernière opposition, comme tu viens de l'indiquer, n'est peut-être ni essentielle, ni véritablement opératoire. Comment alors distinguer l'une de l'autre ces deux sortes de musiciens? Est-ce par le type de «ductions » qu'ils manipulent?

B. S. : Pour penser les différents types de «ductions» que constitue le processus exclamatif en tant qu'il consiste en un circuit du désir, où s'enchaînent les unes aux autres des interprétations qui se font donc écho, que ce soit comme arrangements et dérangements, ainsi que le dit si bien Peter Szendy; ou comme tra-duction entendue comme transmission, et en cela, nécessairement aussi, 
comme transformation, improvisation, variation, etc.; il faut penser les relations entre ces divers types de ductions. Et pour penser ces dernières, il faut utiliser le concept, là encore venu de Simondon, de transduction, c'est-à-dire de relation trans-ductive : une relation qui constitue ses termes.

Le socle classique de nos concepts esthétiques a été forgé depuis le créationnisme et relève en cela d'une conception chrétienne de l'art qui définit le génie comme génération spontanée inspirée par un Dieu lui-même créateur. Mais le XIX ${ }^{e}$ siècle découvre, dans un mouvement qui affecte toutes les études du vivant et de l'humain, que le génie est "génétique », j’entends par là qu'il est inscrit dans un processus qui ne cesse de se trans-former historiquement. En ce moment, je m'attache à montrer que la pensée d'une telle trans - formation requiert une généalogie du sensible, au sens de Nietzsche : il s'agit de généalogie, et non simplement de génétique, dans la mesure où interviennent, dans cette transformation, des facteurs artefactuels, des artifices, des tekhnaï, des traces non vivantes qui affectent de façon essentielle le vivant sensible, de sorte qu'il faut tenir compte en tout premier lieu des dimensions proprement documentaires du processus, de toute cette grisaille des traces (pour parler comme le Nietzsche de la Généalogie de la morale), où s'élaborent les dimensions mnémotechniques du sensible. Je compte les instruments parmi les documents.

C'est tout particulièrement vrai de la musique, où la part organologique des questions et des concepts saute aux yeux des oreilles, si l'on ose dire. Nous, «modernes» ou «contemporains», ne pensons plus le sensible depuis le créationnisme chrétien : nous sommes devenus évolutionnistes, voire postévolutionnistes (j’entends par là une pensée de l'évolution qui intègre l'artificiel comme facteur premier, ainsi que tentent de le donner à sentir certains artistes tels Stelarc ou Orlan). La généalogie du sensible, où doit s'inscrire une pensée renouvelée de l'organo-logie du musical, n'est pas la génétique du vivant : c'est la question d'un "génie » de ce que j'appelle l'épiphylogenèse ${ }^{5}$, c'est-à-dire de ce qui met en œuvre de l'organologique partout, et bien au-delà non seulement du musical, mais même du sensible (il y a par exemple une organologie de l'intellect, et en fin de compte, de toutes les couches de l'existence). L'épiphylogénétique, c'est ce qui articule la mémoire germinale (au sens de Weismann), la mémoire somatique, et la mémoire que constituent les rétentions tertiaires, c'est-à-dire les artefacts quels qu'ils soient. C'est sur la base de ce concept que je développe l'idée d'une organologie générale, non pas au sens de Novalis, encore que celui-ci ait eu sur ce point d'intéressantes intuitions, ni même au sens restreint de Simondon (qui y voit la manière dont les éléments techniques peuvent être conçus comme les organes techniques d'un corps où précisément ils font sinon corps, du moins système), mais au sens où elle
5. Cf. La technique et le temps 1. La faute d'Épiméthée, 1994, et Philosopher par accident, 2004. 
articulerait la psychophysiologie des organes naturels du vivant, la technologie des organes artificiels de la vie comme ex-sistence, c'est-à-dire situation exclamative hors de soi, et la socioanthropologie des organisations caractérisant les différents types de groupements humains.

Dans ce cadre épiphylogénétique, le "génie» interprétatif (je conserve le mot "génie » en tant qu'il désigne un point de singularité littéralement im - probable, incalculable, inespéré au sens d'Héraclite [anelpiston], et cependant inscrit dans la chaîne d'un magnétisme qui, pris au sens du Ion de Platon, interdit de le penser comme pouvoir de création et oblige à l'inscrire dans un circuit auquel, dans la pensée des Tragiques, président les Muses) doit être conçu à partir de différentes instances d'extériorisation selon les possibilités de rétentions tertiaires. Ces dernières forment système, c'est-à-dire entretiennent entre elles des relations transductives telles que les termes de la relation, dans leurs potentialités, ne précèdent pas la relation elle-même; c'est en cela qu'elles surdéterminent l'instanciation de divers rôles interprétatifs. Par « rétentions tertiaires", je ne désigne pas seulement, dans le cas de la musique, les instruments eux mêmes, qui constituent, comme matrices de possibles, des spatialisations de possibilités temporelles et, lorsqu'ils sont joués, des actualisations temporelles à partir de leurs caractéristiques spatiales (ces dernières engrammant aussi cependant des rétentions secondaires collectives et instrumentales). J'entends non seulement cela, donc, mais tout autant les partitions, les disques, les bandes magnétiques, les salles de concert, les dispositifs de diffusion en général, ainsi que les dispositifs de formation des oreilles : celles des musiciens dans les conservatoires et celles des auditeurs via les radios, les organes d'édition et de diffusion, les institutions de programmation musicale (dont l'Ircam par exemple), etc. Tout cela forme un système technique de la musique qui supporte lui-même un systéme social de la musique, et un tel système est une vaste machine interprétative, où chacun joue un rôle dans le circuit de l'exclamation qui est aussi celui du désir de musique.

Penser le système technique de la musique que suppose le système interprétatif d'une époque musicale nécessite, avant même la proposition d'une organologie générale, la constitution d'une organologie élargie, qui permet par exemple de caractériser trans-ductivement ce qui se passe lorsqu'advient la notation : comment la partition va constituer un tenseur du devenir instrumental. Mais on pourrait en donner mille autres exemples : de même que Gui d'Arezzo, croyant mettre au point un système de conservation du patrimoine des chants, faisait une invention dont la Wirklichkeit allait littéralement liquider un certain mode traditionnel de la musique comme interprétation, la phonographie apparue à la fin du XIX siècle, qui était essentiellement conçue comme 
organe d'enregistrement d'une musique constituée par ailleurs, est devenue un élément constituant, au sens phénoménologique, de la réalité de la vie musicale.

Dans un dispositif organologique, ce qui est important, c'est la répétition. Le processus organologique, appuyé sur un dispositif organologique, c'est un ensemble de rétentions tertiaires qui permet la répétition plus ou moins stable d'un événement qui a déjà eu lieu. Cette répétition, quand elle est produite à une époque qui correspond à celle des sociétés archaïques ou traditionnelles, est stabilisée essentiellement par des instruments qui ont pour caractéristique, aussi rudimentaires qu'ils puissent paraître, de rendre possible la répétition d'un son à peu près équivalent à ce que l'on recherche, et, par leur structure physique, de permettre de développer des registres stables correspondant à des rétentions secondaires collectives, bref, de constituer un champ de possibles lié à un instrumentarium ouvrant des possibilités de répétitions de gestes et de sons, gestes d'écriture aussi bien que gestes de performances instrumentales, possibilités de répétitions qui caractérisent donc les époques musicales et à partir desquelles se produisent des différences, c'est-à-dire des possibilités nouvelles qui sont rendues possibles par la combinatoire des répétitions autorisées.

L'écriture musicale est une époque d'un tel processus. Depuis près de dix siècles, la relation transductive entre interprétation par la composition et interprétation par la performance instrumentale constituent les deux pôles principaux du devenir herméneutique en quoi consiste la musique en général, l'inspiration des Muses, celle d'Orphée. Dans le cas des sociétés traditionnelles, ceux qui répètent sont poètes, chanteurs et musiciens, instrumentistes et rhapsodes, c'est-à-dire aussi un peu comédiens; mais c'est aussi le lot des aèdes. (Non seulement il fut un temps où l'instrumentiste et le compositeur n'étaient pas distincts, mais il en fut où la poésie et la musique n'étaient pas distinctes, où la parole ne se distinguait pas de la musique ni n'avait lieu sans instruments : tel est Orphée.) Dans les cas que j’ai cités, un registre se répète sous la forme d'un catalogue que supportent des dispositifs rétentionnels, aussi bien instruments qu'écoles de répétition, généralement initiatiques, inscrites sous les signes d'Éleusis et de Perséphone. La répétition du catalogue donne lieu à une interprétation engendrant une différence dans la répétition. Dès lors que la notation diastématique apparaît, ce catalogue est de plus en plus objectivé, et à partir de cette objectivation, l'interprétation devient non plus simplement interprétation d'un objet temporel par un instrumentiste dans le temps de l'exécution, mais bien interprétation de l'objet spatial qu'est la rétention tertiaire de la partition.

Autrement dit, pour comprendre la différence entre les deux ductions, celle du compositeur et celle de l'instrumentiste, il faut les mettre en relation trans-ductive et pour cela avoir compris qu'elle est ici conditionnée par le 
système que forment la rétention tertiaire instrumentale et la rétention tertiaire diastématique, ce qui nécessite aussi de comprendre ce qui se passe en termes de répétition dans chaque duction, et ce que veut dire repro dans reproduction. La repro-duction signifie que dans les deux cas, il n'y a duction que par reproduction, c'est-à-dire par répétition, selon une voie originale, singulière, propre soit à l'instrument et aux possibilités temporelles contenues dans sa spatialité, soit à la partition, elle aussi espace de figuration et de schématisation du temps (au sens kantien). Mais à leur tour, ces possibilités temporelles propres se «transductent", si l'on peut dire, mutuellement : le compositeur interprète en fonction des possibilités d'interprétation de l'instrumentiste, et ce dernier interprète l'interprétation du fonds de rétentions secondaires collectives formalisées par le compositeur sous forme d'une partititon, tandis que l'un et l'autre ont ce fonds rétentionnel collectif en commun par-delà la spécificité de leurs rôle dans le circuit exclamatif et ses instances organologiques. (Répétition qui veut dire aussi redemande : cette demande suppose des attentes, c'est-à-dire des protentions, celées dans les rétentions et comme leur potentiel de projections singulières.)

Le compositeur met en œuvre des rétentions secondaires collectives qui correspondent à des éléments de la littérature que forme le répertoire et qui deviennent un matériau citationnel que l'on peut ex-citer, activant ainsi un horizon d'attente de la part de l'auditeur. C'est à partir de l'activation de cet horizon d'attente qu'il est paradoxalement possible de produire de l'inattendu : les rétentions secondaires collectives ouvrent des possibles, qui rencontrent, dans certaines conditions, des archi-protentions, c'est-à-dire un matériau qui relève du dionysiaque. J'ai esquissé ce point dans le dernier chapitre de De la misère symbolique 1, et j'y reviendrai plus longuement dans le tome suivant. Ceci fait appel au concept simondonien d'individuation psychique et collective, aussi bien qu'à une relecture de Freud depuis la question de la rétention tertiaire dont je crois qu'il l'a littéralement refoulée, comme toute la tradition occidentale et métaphysique.

Quant à la duction de l'instrumentiste, elle vient retemporaliser ce qui ne peut être que spatial : le travail de la composition, ce n'est que spatial, c'est du temps spatialisé, et en cela, essentiellement en défaut d'être. C'est du virtuel pur. C'est du temps discrétisé et détemporalisé dans cette mesure. Discrétisé, il devient manipulable dans sa détemporalisation temporaire telle que la pratique le compositeur, mais il n'est que virtuel. Il ne peut devenir actuel qu'avec l'interprète, qui doit le re-temporaliser. Or, cette retemporalisation est une sélection primaire dans les rétentions primaires virtuelles en quoi consistent les artifices d'écriture.

Il y a un fantasme du compositeur, qui est de produire une définition spatiale de l'objet temporel musical si précise que celui-ci deviendrait finalement 
intégralement fidèle à sa temporalité virtuelle, c'est-à-dire qu'il équivaudrait point par point dans sa définition spatiale à sa définition temporelle. Mais ce n'est qu'un fantasme : le compositeur peut, certes, lire idéalement la partition, et, dans son oreille interne, l'entendre sonner depuis la visée syn - thétique (com-positionnelle) d'un tout. Ce tout n'est cependant que ce qui, virtuel, consiste à travers la diversité de ses existences possibles, c'est-à-dire de ses occurrences comme interprétations actuelles : littéralement, ce tout, comme virtualité, n'existe pas. Ce qui ex-siste, ce sont les performances instrumentales. Un instrumentiste entend sonner. Mais dans sa manière d'entendre sonner avant même de jouer, il a déjà intégré et intériorisé son instrument dans cette «oreille interne» qui n'est donc pas si interne que cela. De même, le compositeur a intériorisé l'espace diastématique qu'il a parcouru de mille manières en étudiant la composition. Et parce que l'instrumentiste joue la partition mentalement à partir de son savoir musculaire, musculaire, nerveux et corporel en tous sens, corporéité surdéterminée par un savoir musical en général et qui n'est donc pas simplement Körper mais Leib, chair, en ce sens, son savoir n'étant jamais seulement musical, mais psychiquement, affectivement et socialement investi, cet ensemble sapientiel, tramé de rétentions secondaires, collectives ou non, commande son jeu dans l'exécution des rétentions primaires en quoi consiste l'objet temporel que la partition spatialise (et qui n'est donc qu'un objet temporel virtuel).

Les rétentions primaires sont sélectionnées par l'instrumentiste, c'est-à-dire actualisées par lui, selon des modalités d'interprétation chaque fois singulières et qui ne sont pas simplement des effets de ses erreurs de lecture, ou de ses incapacités technico-physiques ou intellectuelles à interpréter exactement ce qui est joué : les sélections en quoi consiste l'actualisation du temps virtuel de la partitition actualisent aussi ses attentes. Ces attentes sont les rétentions secondaires musicales à partir desquelles il peut entendre sonner l'espace et à partir desquelles il peut temporaliser l'espace matriciel qu'est la partition. Cette matrice, en tant qu'elle est répétable, peut produire de la différence : celle en quoi consiste chaque interprétation instrumentale singulière. Cette différence, comme singularité d'un instrumentiste interprète, fait sonner la singularité de la pièce selon une occurrence qu'elle rend possible, mais qui, n'étant que virtuelle, est à la lettre improbable, c'est-à-dire qu'elle ne se réduit pas au résultat d'un calcul, mais mobilise une virtualité protentionnelle beaucoup plus vaste, celle de l'inattendu, de l'inespéré que donne à entendre la musique toujours à nouveau, et comme le nouveau même. Comme le disait Socrate dans Ion, ou plutôt, comme je l'entends et l'interprète dans ce texte, la singularité de la pièce écrite ne peut sonner qu'en faisant sonner la singularité d'un corps 
et d'une âme interprétante lorsque il s'agit d'exécuter cette pièce temporellement sur un instrument. Quant à la duction du compositeur, c'est celle qui entend sonner des pièces dans sa mémoire et qui les fait résonner sous forme de nouvelles pièces écrites et ainsi les exclame. Même si ce n'est pas nécessairement conscient, les classes de composition au conservatoire servent fondamentalement à acquérir une connaissance du répertoire telle que je pourrai le faire résonner, re-sonner, c'est-à-dire le répéter en y extrayant une différence im-probable (... quoique le fruit de savants calculs). C'est ce qui m'intéresse beaucoup dans le travail de Brice Pauset. Mais tous les compositeurs travaillent ainsi, naïvement ou pas.

Bref, la repro-duction est première dans la mesure où c'est depuis sa possibilité que se produisent des transductions, des traductions, etc., éventuellement des trahisons. Il peut y avoir trahison parce que le primat de la repro-duction signifie le défaut absolu et interminable d'une pure duction; cet interminable, qui est aussi la nécessité au sens de Blanchot, c'est ce qui caractérise l'individuation chez Simondon, et ce que Heidegger inscrit dans l'indétermination que constitue l'être-pour-la-mort. Reste que la repro-duction comme originaire défaut d'origine ne se concrétise que comme trame de relations transductives, où une transduction est, par exemple, la relation qui lie l'interprète et le compositeur, relation dans laquelle le compositeur ne précède pas logiquement ou musicalement l'interprète, ne serait-ce que parce que le compositeur est souvent lui-même instrumentiste, et écrit à partir d'un «savoir de l'instrumental» (pour reprendre un mot de Michaël Levinas). C'est à partir d'une compétence d'interprétation instrumentale minimale, les compositeurs ont longtemps composé au piano, que le transducteur qu'est le compositeur spatialise sur la partition qu'il écrit. Ce sont ici la partition et l'instrument de travail qui forment eux-mêmes une relation transductive.

Entre les instances de duction qui s'articulent et qui aujourd'hui sont triples (compositeur, interprète et auditeur), il y a toutes sortes de relations transductives. Ces relations transductives ne peuvent être pensées qu'à partir de la question d'une reproduction originaire qui affecte les trois instances, et cette fois-ci je fais apparaître aussi l'auditeur parce qu'aujourd'hui, celui-ci peut répéter ses auditions à travers les enregistrements. C'est une nouveauté extrêmement importante : avant 1877, un objet temporel musical ne pouvait en aucun cas se reproduire deux fois à l'identique, ni être répété par quelqu'un qui ne le jouerait pas avec un instrument.

Si la reproduction est essentielle dans la différence des ductions à travers les différentes transductions, c'est parce qu'il y a des attentes elles-mêmes constituées par des rétentions secondaires collectives. Les catalogues sont des rétentions 
secondaires collectives d'un certain type, dites de traditions orales, même si en réalité il n'y a jamais seulement de l'oralité, il y a aussi des instruments, diverses fixations tertiaires, dont font aussi partie, par exemple, le churinga et le mythogramme en général. La partition, c'est une autre manière de produire des rétentions secondaires collectives partageables par des interprètes instrumentistes qui vont pouvoir en donner des performances diverses (et il y aurait tout une question de la performativité instrumentale à élaborer ici, je prends le mot au sens de Austin), et par des compositeurs qui vont pouvoir les interpréter par d'autres formes de composition : chaque fois, ces rétentions secondaires collectives engendrent des productions nouvelles de rétentions primaires qui sont elles-mêmes filtrées par des rétentions secondaires collectives. À travers ces sélections secondaires se constitue un horizon la plupart du temps inconscient, qui devient une espèce de seconde nature que l'on ne voit pas, que l'on n'entend pas, mais qui est là, et qui est le matériel repro-ductible avec lequel on peut travailler : c'est un complexe organologique puisque, comme je le disais en commençant, depuis les os percés qui servent de flûtes jusque aux machines numériques les plus perfectionnées, en passant par les disques, les sonnailles de Schaeffner, et tant d'autres choses comme les corps, les organes vocaux, les conservatoires de musique, les radios, tout cela forme un système technique qui surdétermine les questions de reproduction en constituant des transductions. L'essentiel tient au fait que, bien sûr, ce système de repro-duction est un terrain de lutte, voire de guerre. Aujourd'hui, nous vivons une véritable guerre esthétique : il s'agit d'imposer les rétentions secondaires collectives qui conviennent à la machinerie des industries culturelles et aux fonctions qu'elle sert, à savoir la socialisation consensuelle des produits de la société hyperindustrielle. Penser ce conflit devrait être le programme d'une politique de la repro-duction ${ }^{6}$.

Tout cela n'a de sens que parce que la musique est textuelle en tant que trame de rétentions qui forment un tissu, et ce tissu textuel demande toujours interprétation. Le tissu textuel, musical ou autre, c'est ce qui indexe une modalité du défaut d'origine de l'humanité en général, selon une époque qui est propre à l'Occident, et comme discrétisation, qu'il s'agisse du flux temporel des paroles (alphabet) ou de celui des arpèges et des harmonies (diastemata et portée). L'interprète d'un texte musical, que ce soit un compositeur ou un instrumentiste, c'est celui qui accuse le défaut, c'est-à-dire qu'il le désigne, le met en cause, mais aussi, qui est mis en cause par lui. Ce défaut, c'est ce qu'il faut. Il est très intéressant de voir que le programme CHANT réalisé par Xavier Rodet au début de l'histoire de l'Ircam, qui réalisait une synthèse de voix, était inécoutable parce que la voix de synthèse n'avait aucun défaut. On sait aujourd'hui,
6. Cela constitue l'objet principal de De la misère symbolique, et c'est ce que désigne, au bout du compte, le concept deleuzien de sociétés de contrôle, cf. De la misère symbolique 1, premier chapitre en particulier. 
7. Kontra-Sonate (1999-2000), enregistrement par Andreas Staier chez Aeon (AECD0421). y compris dans les logiciels non artistiques, par exemple dans le monde financier, que l'on est obligé d'ajouter artificiellement de l'aléa et du défaut pour conférer une présence ou une crédibilité au matériel synthétique. La textualité, en tant qu'elle fait défaut, porte le défaut et fait sonner le défaut, la textualité de la musique ainsi entendue est irréductible dans la mesure où un texte musical ne s'interprétera jamais autrement qu'à travers la singularité d'un contexte qui est à la fois l'interprète, l'auditeur avec ses rétentions secondaires spécifiques, la salle, la spécificité de l'instrument ou du corps instrumental, l'ensemble instrumental mis en œuvre, etc. C'est l'una volta, l'unique fois que, certes, la reproductibilité technique peut justement rendre à son tour interprétable parce que répétable : il ne s'agit pas du tout d'opposer l'unique fois à sa répétition, mais, bien au contraire, de montrer que cette unique fois n'a lieu qu'en faisant défaut, et en appelant sa répétition, telle qu'elle ne peut que donner, à son tour, une nouvelle différence. C'est ce que Deleuze a pensé dans Différence et répétition, mais c'est aussi la « différance » au sens de Derrida.

Ce qui fait l'autorité d'un interprète, que ce soit un compositeur, un chef d'orchestre ou un instrumentiste, c'est sa capacité à produire des sélections primaires dans le matériau des rétentions primaires, des sélections primaires qui soient pertinentes, et qui deviennent, de fait ou de droit, des rétentions secondaires collectives. C'est ce qui fonde l'autorité de l'interprète. C'est aussi vrai des interprètes que sont avant tout autre rôle un imam, un rabbin ou un curé. Et c'est aussi ce qui se passe dans l'histoire de la peinture : Cézanne et tant d'autres copient, c'est-à-dire interprètent, c'est à partir de cette interprétation qu'ils peignent et que Cézanne pouvait passer «sur le motif» : il s'agit dès lors de "lire et interpréter la nature », disait-il. Mais c'est à partir de sa scrutation interprétative qui s'exclamait d'abord en copiant au musée du Louvre qu'il pouvait lire et interpréter sur le motif. Le compositeur aussi est celui qui produit des sélections primaires qui se transforment en rétentions secondaires collectives, comme on le voit dans ce que fait Bartók de la musique populaire. C'est encore cette question que visent Pauset et Staier dans l'interprétation de la Kontra-Sonate ${ }^{7}$ où Staier demande à Pauset pourquoi il a tellement noté d'éléments de dynamique dont l'interprète ne peut pas faire grand-chose, parce qu'il y en a trop. Pauset répond que c'est pour intensifier la question de l'interprétation. Ce n'est pas pour que l'instrumentiste joue toutes les indications : il s'agit de tendre ou bander en lui la singularité de son interprétation.

Il y a aussi une interprétation de l'auditeur, de l'auditeur qui, aujourd'hui, ne sait ni écrire la musique ni jouer d'un instrument, mais qui peut répéter son écoute et, en cela, discriminer et interpréter, tandis qu'il interprète aussi la musique dans ou sur d'autres registres que le musical : il transpose dans ses 
propres registres, y compris dans sa vie quotidienne affective, intime ou publique, par exemple en dansant.

Si diverses et différentes qu'elles puissent être, les interprétations de l'instrumentiste, du compositeur ou de l'auditeur sont à la fois complémentaires, indissociables parce que mutuellement «transduites», et solidairement exclamatives. La situation de repro-duction induit l'exclamation, et l'exclamation signifie que quand je reçois un affect à travers une œuvre musicale ou un donné esthétique quelconque, je ne le reçois que dans la mesure où je me prépare à le rendre. C'est une économie du don, c'est un circuit : le circuit du désir. Comme circuit de l'exclamation, l'économie libidinale en quoi ce désir consiste et se constitue est un faire-signes : c'est une production de symboles. Il y a différentes modalités de l'exclamation, et l'exclamation se prépare : un interprète instrumentiste qui répète prépare sa capacité exclamative dans la répétition instrumentale. Un compositeur s'exclame de ce qu'il a entendu sous la forme de la répétition graphiquement spatialisée de ce qu'il entend. Un auditeur exclame en transposant sur un autre registre, au minimum en applaudissant ou en huant. Dans les trois cas, il s'agit d'un faire-signes, plus ou moins riche, et toujours, finalement, en symbolisant à proprement parler, parfois beaucoup plus tard, fût-ce comme simple bavardage après le concert, misère symbolique qui est le lot courant de la nécessité du faire-signes ne se manifestant que par son défaut (ce dernier devenant alors un manque : le manque de jugement).

L'exclamation est donc le processus du faire-signes, c'est-à-dire du signifiant en tant que ce qui ouvre la possibilité de la différence entre signifiant et non insignifiant.

N. D. : Tu citais tout à l'heure la trahison comme cas limite (ou marginal?) des transductions, traductions, etc. en quoi consiste la repro-duction. Que la trahison soit une bonne ou une mauvaise exclamation, ce qui est sûr du moins, c'est qu'elle en provoque d'autres. Par exemple celles de Platon dans La République, pour reprendre un exemple que tu as un jour évoqué lors d'un séminaire à l'Ircam, en comparant la façon dont l'interprète est verrouillé (voire renié) par une certaine lignée musicale avant-gardiste au $\mathrm{XX}^{\mathrm{e}}$ siècle, avec la façon dont le poète, tombé sous l'accusation de haute trahison, est chassé de la Cité platonicienne.

B. S. : Le fait est que la notion de trahison sert souvent à distinguer la spécificité de l'interprétation parmi les différents modes exclamatifs que tu viens de mettre en relation les uns avec les autres. Est-ce parce que la frontière entre repro-duction et trahison est plus poreuse dans l'interprétation que dans les autres cas? Et si c'est bien le cas, la trahison relève-t-elle alors d'une forme de nécessité? 
On pose toujours, et, d'une certaine façon, nécessairement avec raison, que le traducteur est un traitre (traduttore traditore comme on dit en italien). Cette traîtrise en quelque sorte inévitable est la marque du défaut d'origine. Évidemment, il y a plusieurs types de traîtrises, par exemple celle dont Antoine Berman parle dans L'épreuve de l'étranger, à propos de Voltaire qui « arrange » des textes de Shakespeare parce qu'ils ne lui conviennent pas. C'est alors une trahison pure et simple.

Il y a une histoire de la trahison comme trans-duction/tra-duction qui est extrêmement complexe, et qui est sous-tendue par toute une tradition de négociations avec son irréductibilité, avec ce qu'il faut appeler sa né-cessité. La relation entre trahison, traduction, tradition et transmission impose des règles qui changent avec le temps selon les changements de rétentions tertiaires. Typiquement, dans une société où il n'y a pas de notation, il ne peut y avoir que de la «trahison », c'est-à-dire de la trans-formation, mais par le fait même qu'il n'y a pas de texte établi comme tel, il ne saurait y avoir de «trahison». Et dans tous les cas, même quand il y a une notation que j'appelle orthothétique (relevant des modes d'enregistrement ou de transmission orthothétiques, c'est-à-dire qui posent [thésis] exactement ou droitement [orthos], l'interprétation est nécessairement une sélection actuelle qui induit une altération du virtuel à travers sa diachronisation. Jouer une pièce de musique, c'est pour moi la "parler ", c'està-dire la diachroniser dans un rapport de l'interprétation à l'interprété qui ressemble au rapport de la parole à la langue tel que Saussure définissait celle-ci comme synchronie.

Entre traduction et transduction, le lien est évident : je pense de fait l'interprétation comme une tra-duction, très proche, je crois, de la pensée de Berman. Le traducteur est lui aussi un sélecteur parmi des rétentions primaires possibles, qu'il soumet à un certain esprit de sa propre langue, précisément en tant qu'elle est tramée de rétentions secondaires collectives supportant des attentes et formant un «esprit». Mais une bonne traduction ne se réduit pas à cela : c'est une écriture, une composition, l'occurrence d'une singularité dans une autre singularité. Quiconque a un jour rencontré l'étrangeté d'un poème aimé de lui ou d'elle dans une langue étrangère le sait. Une fois cela posé, il peut y avoir de mauvaises traductions, au sens de trahisons à proprement parler, qui ignorent délibérément la nécessité de l'étranger, précisément, qui l'acclimatent au point de le réduire à rien, ainsi de Voltaire «traduisant» Shakespeare. Quant au rejet de l'interprétation par Platon, il procède du rejet du corps aussi bien que de la tekhnè, des hypomnémata de l'écriture et de ce que la dissémination des textes, pour reprendre un mot et un titre de Derrida, emporte d'incontrôlabilité des singularités, c'est-à-dire, aussi bien, d'inachèvement de toute lecture et de toute 
écriture, du fait, pour le dire encore autrement, que l'interprétation est interminable et improbable, restant à jamais sous la forme d'une promesse. Cela veut dire d'ailleurs aussi que toute interprétation, comme toute traduction, est toujours d'une manière quelconque une trahison, en ce qu'elle est nécessairement finie : il ne saurait y avoir de traduction satisfaisante, un poème ne peut être lu que dans sa langue d'origine. Et c'est vrai aussi de toute interprétation, à ceci près que ce qui est vrai de la traduction inter-langues est aussi vrai de la lecture comme traduction intralinguistique (puisqu'une langue est constituée de paroles et d'idiomes tout aussi étrangers les uns aux autres, en fin de compte, que les langues dites «étrangères»), et que finalement, toute transduction induit en tout cas, comme relation dynamique, le jeu d'une inadéquation qui, justement, donne du jeu, et l'envie de jouer : c'est encore le circuit du désir comme don et contre-don.

Dès lors cependant qu'il s'agit de penser à partir de la distinction, historiquement située, entre instrumentiste et compositeur, qui a induit une opposition erronée, métaphysiquement constituée à partir de la péjoration de l'interprétation par Platon, entre écriture et interprétation, de telles questions doivent être abordées comme des expressions historiques du processus de grammatisation qu'a décrit Sylvain Auroux à propos de la discrétisation du linguistique, et que je tente de mobiliser dans le domaine du musical à côté de la question grammatologique de Derrida. Ce concept de processus de grammatisation est en effet particulièrement intéressant pour analyser le sens de la discrétisation diastématique en musique. De même que la question de la traduction et de l'interprétation ne peuvent se poser en tant que telles dans le domaine de la poésie et plus généralement du langage que lorsque celui-ci est grammatisé par l'alphabet (qui rend possibles des questions et des pratiques comme celle de la traduction, aussi bien que la constitution du texte comme objet identique d'interprétations variées, ouvrant par là même la possibilité de disciplines comme la grammaire, la rhétorique et la poétique), il faut attendre la grammatisation du temps musical, c'est-à-dire sa spatialisation, pour qu'adviennent nos questions d'interprétation. Dans tous les cas, la tentation est grande de confondre la rigueur et même l'exactitude (orthotès) de l'engrammage des intentions linguistiques ou musicales par les textes grammatisés, avec la possibilité de supprimer l'interprète. Mais c'est là ne rien comprendre à ce que c'est qu'un texte, à savoir un support rétentionnel tertiaire, qui permet la reconstitution d'un flux primaire, consistant lui-même en sélections toujours à la fois singulières et finies. 


\section{BIBLIOGRAPHIE}

ARTAUd, A. (2003) Pour en finir avec le jugement de Dieu, Paris, Gallimard, coll. «Poésie», 230 p. Auroux, S. (1995) (s. la dir. de), La révolution technologique de la grammatisation, Liège, Mardaga, coll. «Philosophie et Langage ", $224 \mathrm{P}$.

Berman, A. (1984), L'épreuve de l'étranger : culture et traduction dans l'Allemagne romantique : Herder, Goethe, Schlegel, Novalis, Humboldt, Schleiermacher, Hölderlin, Paris, Gallimard, coll "Les Essais", 311 p.

Deleuze, G. (1968), Différence et répétition, Paris, Presses Universitaires de France, coll. «Épiméthée ", $409 \mathrm{p}$.

Dufourt, H. (1981), "L'artifice d'écriture dans la musique occidentale», Critique n ${ }^{\circ} 408$ (mai 1981) [L'œil et l'oreille. Du conçu au perçu dans l'art contemporain], p. 465-477.

KLEE, P. (1964), Théorie de l'art moderne (trad. fr. par P.-H. Gonthier), Genève, Gonthier, coll. "Médiations", 1964, $174 \mathrm{P}$.

LoRTAT-JaCOB, B. (1998), Chants de passion. Au cœur d'une confrérie de Sardaigne, Éditions du Cerf, 352 p., CD.

SCHAEFFner, A. (1936), Origine des instruments de musique. Introduction ethnologique à l'histoire de la musique instrumentale, Paris, Payot, $405 \mathrm{P}$.

Simondon, G. (1989), Lindividuation psychique et collective : à la lumière des notions de forme, information, potentiel et métastabilité, Paris, Aubier, coll. «L'invention philosophique », $293 \mathrm{P}$.

SzENDY, P. (2000), (s. la dir. de), Arrangements, dérangements : la transcription musicale aujourd'hui, Paris, Ircam/L'Harmattan, coll. «Les cahiers de l'Ircam », $139 \mathrm{p}$. 